\title{
Commentary: Cardioplegia solutions for myocardial protection-Dealer's choice
}

\author{
Zaid Abdelsattar, MD, MS, and Juan A. Crestanello, MD
}

\author{
From the Department of Cardiovascular Surgery, Mayo Clinic, Rochester, Minn. \\ Disclosures: Authors have nothing to disclose with regard to commercial support. \\ Received for publication March 28, 2019; accepted for publication March 28, 2019; available ahead of print May \\ $15,2019$. \\ Address for reprints: Juan A. Crestanello, MD, Department of Cardiovascular Surgery, Mayo Clinic, 200 First St \\ SW, Rochester, MN 55905 (E-mail: crestanello.juan@mayo.edu). \\ J Thorac Cardiovasc Surg 2020;159:1864-5 \\ $0022-5223 / \$ 36.00$ \\ Copyright (c) 2019 by The American Association for Thoracic Surgery \\ https://doi.org/10.1016/j.jtcvs.2019.03.114
}

In this issue of the Journal, Vivacqua and colleagues ${ }^{1}$ report the results of their randomized trial and demonstrate the noninferiority of a single dose of Custodiol HTK (Essential Pharmaceuticals, LLC, Durham, NC) relative to repetitive cold blood cardioplegia in protecting the myocardium, as measured by postoperative cardiac enzyme leaks. This study adds to the growing literature comparing different types of solutions for myocardial protection.

Myocardial protection is a multifaceted stepwise process. It depends not only on the type of solution used but also on 3 other factors in this simplified conceptual model (Figure 1).

Approach. The approach and temperature of administering cardioplegia, whether it is antegrade, retrograde, or a combination of both, needs to be individually tailored to the patient and operation at hand. ${ }^{2}$ In their current study, Vivacqua and colleagues ${ }^{1}$ individually tailored the approach according to the presence of aortic insufficiency. It is unknown whether coronary artery disease or coronary artery bypass grafting influenced their approach, bearing in mind that coronary artery bypass grafting only accounted for $10 \%$ of the study population.

Solution. Several observational studies, randomized trials, and meta-analyses have attempted to elucidate which cardioplegia solution provides the best myocardial protection. ${ }^{3-5}$ However, the search for the ideal solution continues. The study by Vivacqua and colleagues ${ }^{1}$ demonstrated that a single dose of Custodiol cardioplegia solution was not inferior to traditional blood cardioplegia by measuring postoperative cardiac enzymes. The study of Vivacqua and colleagues ${ }^{1}$ was modeled after a previously published trial from Norway that showed similar results.

Time. Even after administering the perfect cardioplegia solution through the perfect approach, it is important to note that the myocardium is still in a period of relative ischemia. Performing an expeditious, efficient, and technically perfect operation becomes of the utmost importance during this period. Vivacqua and colleagues ${ }^{1}$ suggest that

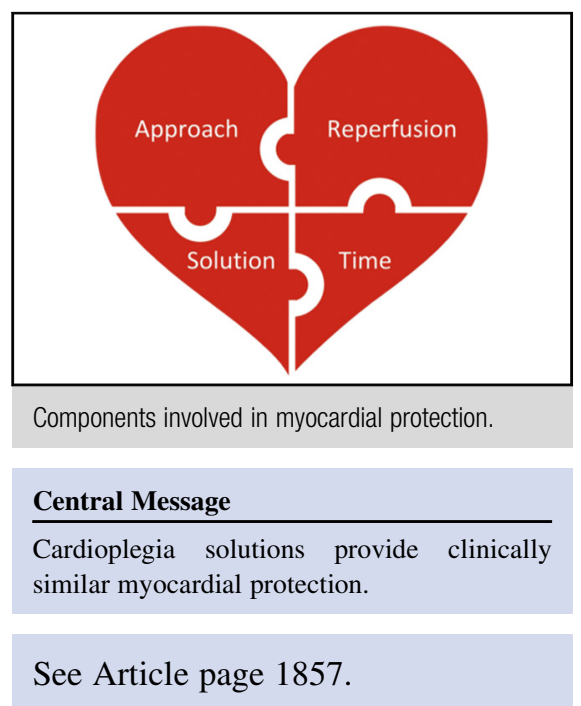

a single dose of cardioplegia is less disruptive and more efficient for the flow of the operation than repeated dosing. Their data, however, show that the crossclamp times were not different between the groups. On the other hand, repetitive dosing could provide the surgeon with a sense of security by actively protecting the myocardium every 20 minutes. In addition, one might argue that the "surgeon's mental break" every 20 minutes necessitated by repetitive dosing is beneficial to reset the 20-minute focused attention span of the human brain. It is commonly agreed on (albeit without much scientific evidence) that the selective sustained attention or focused attention, which is the level of attention that produces consistent results on a task with time, lasts as long as 20 minutes. In other words, the repetitive cardioplegia 20-minute interval interruption does not slow down the operation, and it might actually provide the surgeon with both a sense of security and a mental break to refocus on the task at hand. Neither single nor repetitive cardioplegic administration appears to be detrimental.

Reperfusion. The ischemic myocardium is susceptible to reperfusion injury after the release of the crossclamp. ${ }^{6}$ Many surgeons use a controlled reperfusion strategy with warm cardioplegia or a hot shot to limit reperfusion injury and reduce the incidence of ventricular fibrillation while the patient is coming off bypass. The traditional repetitive blood cardioplegia group in this study did not receive controlled reperfusion. Although the incidence of arrhythmias was similar between the groups, it is unclear whether 


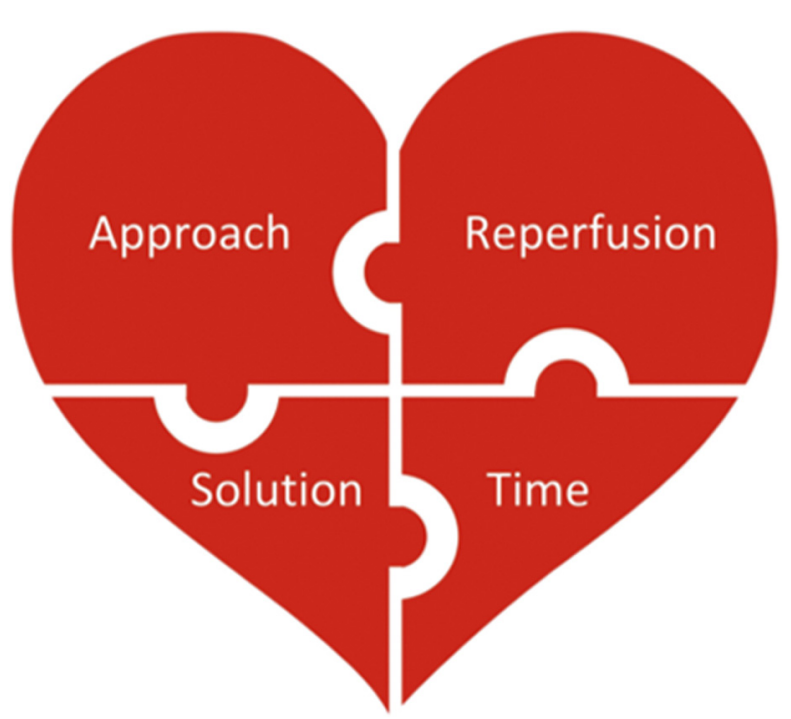

FIGURE 1. Components involved in myocardial protection.

intraoperative arrhythmia rates were different. Custodiol HTK solution is hypothesized to be associated with a lower incidence of reperfusion injury because of the presence of histidine.

\section{CONCLUSIONS}

The study by Vivacqua and colleagues ${ }^{1}$ has several limitations. The power calculation that was performed was not congruent with their primary outcome of interest. The groups remained heterogeneous, with underrepresentation of myocardial revascularization operations in the Custodiol group. Finally, the majority of the patients in this study had preserved ejection fraction. Whether any differences in myocardial protection exist between traditional repetitive cardioplegia and single-dose cardioplegia in patients with low ejection fraction undergoing coronary artery bypass grafting remains unknown. Notwithstanding these limitations, Vivacqua and colleagues ${ }^{1}$ are to be commended on conducting this thoughtful trial. It is especially challenging to perform well-conducted noninferiority trials, ${ }^{7}$ especially in the field of cardiac surgery.

Generally speaking, there are 3 missions in cardiac surgery to ensure good outcomes: protect the heart, protect the brain, and perform a technically good operation. To protect the heart, the cardioplegia solution remains a dealer's choice.

\section{References}

1. Vivacqua A, Robinson J, Abbas AE, Altshuler JM, Shannon FL, Podolsky RH, et al. Single-dose cardioplegia protects myocardium as well as traditional repetitive dosing: a noninferiority randomized study. J Thorac Cardiovasc Surg. 2020; 159:1857-63.e1.

2. Buckberg GD. Antegrade cardioplegia, retrograde cardioplegia, or both? Ann Thorac Surg. 1988:45:589-90.

3. Braathen B, Jeppsson A, Scherstén H, Hagen OM, Vengen Ø, Rexius H, et al. One single dose of histidine-tryptophan-ketoglutarate solution gives equally good myocardial protection in elective mitral valve surgery as repetitive cold blood cardioplegia: a prospective randomized study. J Thorac Cardiovasc Surg. 2011;141: 995-1001.

4. Guru V, Omura J, Alghamdi AA, Weisel R, Fremes SE. Is blood superior to crystalloid cardioplegia? A meta-analysis of randomized clinical trials. Circulation. 2006;114(1 Suppl):I331-8.

5. Mick SL, Robich MP, Houghtaling PL, Gillinov AM, Soltesz EG, Johnston DR, et al. del Nido versus Buckberg cardioplegia in adult isolated valve surgery. $J$ Thorac Cardiovasc Surg. 2015;149:626-34; discussion 634-6.

6. Buckberg GD. Controlled reperfusion after ischemia may be the unifying recovery denominator. J Thorac Cardiovasc Surg. 2010;140:12-8. e1-2.

7. Mauri L, D'Agostino RB Sr. Challenges in the design and interpretation of noninferiority trials. N Engl J Med. 2017;377:1357-67. 